CAMILA MATHIAS DOS SANTOS

\begin{abstract}
AVALIAÇÃO DAS PROPRIEDADES IMUNOMODULADORAS DE TOXINAS TERMO-LÁBEIS DO TIPO II PRODUZIDAS POR Escherichia coli ENTEROTOXIGÊNICA (ETEC) ADMINISTRADAS POR VIA TRANSCUTÂNEA
\end{abstract}


CAMILA MATHIAS DOS SANTOS

\section{AVALIAÇÃO DAS PROPRIEDADES IMUNOMODULADORAS DE TOXINAS TERMO-LÁBEIS DO TIPO II PRODUZIDAS POR Escherichia coli ENTEROTOXIGÊNICA (ETEC) ADMINISTRADAS POR VIA TRANSCUTÂNEA}

Dissertação apresentada ao Departamento de Microbiologia do Instituto de Ciências Biomédicas da Universidade de São Paulo, para obtenção do Título de Mestre em Ciências (Microbiologia).

Orientador: Prof. Dr. Luís Carlos de Souza Ferreira

São Paulo 


\section{RESUMO}

MATHIAS-SANTOS, C. Avaliação das propriedades imunomoduladoras de toxinas termo-lábeis do tipo II produzidas por Escherichia coli enterotoxigênica (ETEC) administradas por via transcutânea. 2009. Dissertação (Mestrado em Microbiologia) - Instituto de Ciências Biomédicas, Universidade de São Paulo, São Paulo, 2009.

As enterotoxinas termo-lábeis (LT-I, LT-Ila e LT-Illb) expressas por Escherichia coli enterotoxigênica são potentes adjuvantes sistêmicos e de mucosa, capazes de aumentar ou modular a resposta imune contra antígenos coadministrados. Essas proteínas são moléculas heterohexaméricas do tipo $A B_{5}$, compostas por uma subunidade $A$ enzimaticamente ativa e um pentâmero $B$, responsável pela ligação as células alvo. Embora estruturalmente semelhantes LT-I e LT-Ila compartilham baixa identidade $(<14 \%)$ em suas subunidades $B$ e afinidade de ligação a receptores distintos, características que podem resultar em propriedades imunológicas diferenciais. O principal objetivo deste trabalho foi avaliar a resposta imune induzida pela toxina LT-Ila e seu pentâmero B (LT-IlaB) empregando duas vias de inoculação: as vias transcutânea e intradérmica. As proteínas recombinantes foram super expressas em linhagens de $E$. coli , purificadas por cromatografia de afinidade e avaliadas quanto à funcionalidade in vitro. Numa segunda etapa, as propriedades imunomoduladoras de LT-Ila e LT-IlaB, com ênfase na imunogenicidade e atividade adjuvante, foram avaliadas em modelo murino. A resposta imune humoral e celular induzida contra a proteína ovalbumina (OVA), aplicada como antígeno nos ensaios de imunização, foi determinada. Os resultados obtidos demonstram que as atividades adjuvantes induzidas por LT-Ila e LT-I variam de acordo com a via de inoculação e que a administração intradérmica dessas toxinas induz respostas inflamatórias distintas.

Palavras-chave: Escherichia coli enterotoxigênica; toxina termo-lábil; adjuvantes vacinais; imunização transcutânea; imunização intradérmica; ovalbumina. 


\begin{abstract}
MATHIAS-SANTOS, C. Evaluation of The immunomodulatory properties of type II heat-labile toxins produced by enterotoxigenic Escherichia coli (ETEC) administered by transcutaneous route. 2009. Master thesis (Microbiology) Instituto de Ciências Biomédicas, Universidade de São Paulo, São Paulo, 2009.
\end{abstract}

The heat-labile enterotoxins (LT-I, LT-Ila and LT-Ilb) expressed by enterotoxigenic Escherichia coli are potent systemic and mucosal adjuvants able to increase or modulate the immune response against co-administered antigens. These proteins are type $\mathrm{AB}_{5}$ heterohexameric molecules, comprising an enzymatically active A subunit and an B pentamer, responsible for binding the target cells. Although structurally similar, LT-Ila and LT-I share low identity values (<14\%) for their B subunits and display different affinity to cellular receptors which might impact their immune modulation activities. The main objective of this study was to evaluate the immune responses induced by toxin LT-Ila and its B subunit (LT-IlaB) using two different inoculation routes: the transcutaneous and intradermal routes. The recombinant proteins were over expressed in $E$. coli strains, purified by affinity chromatography and evaluated for functionality in vitro. In a second moment, the immunomodulatory properties of LT-Ila and LT-IlaB, with emphasis on the immunogenicity and adjuvant activities, were evaluated in murine model. The humoral and cellular immune responses induced against the protein ovalbumin (OVA), used as antigen in immunization assays, was determined. The results show that the adjuvant activity induced by LT-Ila and LT-I differ, according to the route of inoculation, and that the intradermal administration of these toxins induces different inflammatory responses.

Key words: enterotoxigenic Escherichia coli; vaccine adjuvants; heat-labile toxin; transcutaneous immunization; intradermic immunization; ovalbumin. 


\section{INTRODUÇÃO}

Desde a invenção da vacina contra a varíola, ocorrida no ano de 1789 por Edward Jenner, formulações vacinais representam a estratégia com melhor custo benefício descoberta para o controle profilático de doenças infecciosas. Muitas doenças responsáveis por milhões de óbitos no passado (sobretudo entre crianças e idosos) estão hoje erradicadas, como a varíola, ou em vias de desaparecer, como a poliomielite, o sarampo, a coqueluche, a difteria, entre outras, conseqüência direta da descoberta, produção e aplicação generalizada de vacinas. No entanto, o problema das doenças infecciosas ainda persiste e representa uma ameaça real para toda a humanidade. Atualmente, entre os maiores desafios no campo da vacinologia incluem-se 0 desenvolvimento de vacinas com propriedades terapêuticas e a busca por novos adjuvantes, eficazes e seguros para emprego em animais e principalmente, em humanos.

O sistema imune inato é capaz de reconhecer porções antigênicas presentes na superfície de patógenos, conhecidas como PAMPs (padrões moleculares associados à patógenos) (BIANCHI, 2007). O objetivo de uma formulação vacinal é apresentar esses antígenos ao organismo, de maneira inofensiva e controlada, para geração de resposta imune adequada e duradoura. Vacinas baseadas em microrganismos vivos são em geral imunogênicas, entretanto o risco de reversão parcial ou total de virulência tem encorajado o emprego de proteínas purificadas como antígenos vacinais. Embora mais seguras, as vacinas de subunidades são pouco imunogênicas, gerando uma fraca resposta imune, associada principalmente à natureza das moléculas utilizadas, em geral, proteínas recombinantes. Nesse contexto surge a necessidade do uso de adjuvantes vacinais, moléculas capazes de aumentar ou modular a resposta imune a um determinado antígeno (DOUGAN e HORMAECHE, 2006).

Os adjuvantes aprovados atualmente para uso em humanos, a saber: os sais de alumínio, duas emulsões óleo em água e um agonista de TLR (do inglês Toll-like receptors) (DE GREGORIO et al., 2008) não contemplam as necessidades de muitas formulações vacinais em desenvolvimento. Debelar patógenos intracelulares, por exemplo, requer a indução de resposta imune celular, com presença de linfócitos T citotóxicos (LTC), praticamente ausente quando sais de alumínio, conhecidos por 
induzirem fortes respostas de anticorpos, são empregados como adjuvantes. É importante ressaltar ainda a importância de outros fatores, como a natureza do antígeno utilizado e a via de administração na geração de resposta imune adequada.

A pesquisa na área de novos adjuvantes visa desenvolver produtos eficazes e seguros para uso em animais e humanos. Embora muitas substâncias apresentem algum tipo de atividade adjuvante, entre as quais podemos citar: micropartículas, lipossomos, motivos CpG e diversos componentes bacterianos (LPS, flagelina, porções da parede celular e toxinas), grande parte desses compostos não pode ser empregada em formulações vacinais pois apresenta também toxicidade.

Além da capacidade de aumentar a resposta imune contra um determinado antígeno, outra característica importante de adjuvantes vacinais é a capacidade de modulação da resposta imune, possibilitando a geração de respostas adequadas ao combate ou eliminação do patógeno-alvo. Entre as classes de adjuvantes com atividade imunomoduladora em estudo, destacam-se as enterotoxinas termo-lábeis produzidas por Escherichia coli enterotoxigênica (ETEC) e Vibrio cholerae denominadas LTs e CT, respectivamente.

Esses fatores de virulência são toxinas do tipo $A B_{5}$, compostas por uma subunidade A enzimaticamente ativa com peso molecular de $28 \mathrm{kDa}$ e uma subunidade $B$ pentamérica de $58 \mathrm{kDa}$ responsável pela ligação às células alvo por meio de interação com receptores específicos (Figura 1) (MERRITT e HOL, 1995). A liberação dessas toxinas durante a infecção do trato gastrointestinal leva a um quadro clínico de diarréia aquosa profusa. Essas proteínas exercem seus efeitos tóxicos sobre os enterócitos após a internalização, mediada pela interação entre a subunidade $B$ pentamérica e receptores presentes na superfície das células-alvo. Após alcançar o citoplasma, a subunidade A catalisa a transferência de um grupo ADP-ribose à porção $\alpha$ da proteína G estimulatória, levando em última instância a ativação da enzima adenilato ciclase com conseqüente aumento nos níveis intracelulares de $\mathrm{AMPc}$, causando a inibição da absorção dos íons sódio com excreção de íons cloreto e líquido para o lúmen intestinal, o que desencadeia o quadro clínico de diarréia (RAPPUOLI et al., 1999). A afinidade a receptores específicos presentes na superfície de células suscetíveis é determinada pelo pentâmero de subunidades B (FUKUTA et al., 1988). 


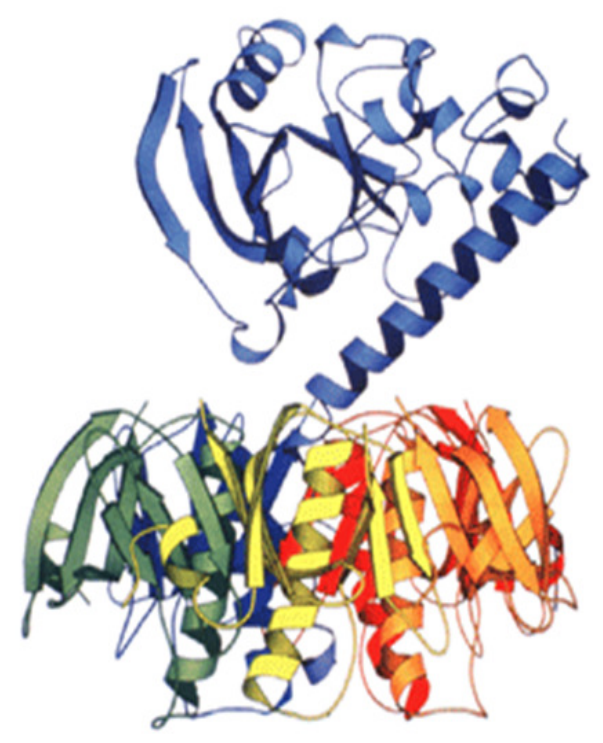

Figura 1. Estrutura cristalográfica da toxina termo-lábil (LT). A proteína é composta por uma subunidade $A$ enzimaticamente ativa (apresentada em azul) que se liga de maneira não covalente a um pentâmero de subunidades $B$, responsável pela ligação a receptores presentes na superfície das células-alvo (Modificado de SALMOND et al., 2002).

As enterotoxinas termo-lábeis são classificadas em dois grupos principais com base em características genéticas, bioquímicas e imunológicas e que não apresentam reação imunológica cruzada (GUTH et al., 1986a; HAJISHENGALLIS et al., 2005a). O grupo I inclui a toxina termo-lábil (LT-I) produzida por algumas linhagens de E. coli, a toxina colérica (CT) expressa por $V$. cholerae e toxinas antigenicamente relacionadas produzidas por outras bactérias entéricas Gramnegativas (HAJISHENGALLIS et al., 2005a). A toxina termo-lábil do tipo I de ETEC (LT-I), comumente conhecida como LT, expressa por linhagens de $E$. coli patogênicas para humanos e animais é muito semelhante à $\mathrm{CT}$, com a qual compartilha estrutura, afinidade ao receptor GM1 em células eucarióticas e atividade enzimática (SPANGLER, 1992). No entanto, estas toxinas exibem diferenças em relação aos mecanismos de regulação gênica, ao seu processamento, secreção e no padrão de resposta imunológica induzida (NATARO e KAPER, 1998; SÁNCHEZ e HOLMGREN, 2005). Os genes etxA e etxB que codificam para as subunidades $A$ e $B$ de $L T$, respectivamente, estão organizados em óperon e são encontrados naturalmente em plasmídeos de alto peso molecular presentes em linhagens de ETEC (SMITH e LINGGOOD, 1971; GYLES et al., 1977).

Ao contrário de CT, que se liga preferencialmente a GM1, um gangliosídeo presente em células nucleadas de mamíferos, incluindo células epiteliais presentes na pele e mucosas além de linfócitos e células dendríticas (DCs) (ANJUÈRE et al., 
2003), LT se liga com diferentes graus de afinidade a outras moléculas inseridas na membrana das células alvo, como por exemplo, asialo-GM1, GD1b, outros glicoesfingolipídeos, receptores glicoproteicos, poliglicosilceramidas (PGC), paraglobosídeos e galactoproteínas (WILLIAMS; HIRST; NASHAR, 1999; FUKUTA et al., 1988). Essa diferença de afinidade a receptores parece estar relacionada às diferenças na seqüência gênica das proteínas, que apresentam $80 \%$ de identidade em suas seqüências primárias.

O segundo grupo de enterotoxinas termo-lábeis compreende as toxinas termo-lábeis do tipo II (LT-II) produzidas por linhagens de ETEC isoladas de animais, produtos alimentícios e raramente de humanos sintomáticos ou assintomáticos (GUTH et al., 1986b; SERIWATANA et al., 1988). Diferente do observado para LT-I, cujo óperon etx se apresenta em plasmídeos, os genes que codificam para as toxinas LT-II são cromossomais (GREEN et al., 1983). Este grupo de LT, descrito na década de 80 , compartilha de 55 a $57 \%$ de identidade em relação à subunidade $A$ de CT e LT-I, respectivamente, e baixa homologia (<14\% de identidade) em relação à subunidade $\mathrm{B}$ das mesmas toxinas (PICKETT et al., 1987). Dois variantes antigênicos de LT-II, denominados LT-Ila e LT-Ilb, que apresentam reação imunológica cruzada foram caracterizados e apresentam 71 e 66\% de homologia entre suas subunidades A e B respectivamente (PICKETT et al., 1989). LT-Ila liga-se preferencialmente ao gangliosídeo GD1b, sendo capaz de se ligar com menor afinidade aos gangliosídeos GD1a, GM1, GT1b, GQ1b e GD2, enquanto LT-Ilb ligase com alta afinidade a GD1a e com baixa afinidade ao gangliosídeo GT1b (FUKUTA et al., 1988). As toxinas LT-Ila e LT-Ilb produzidas respectivamente pela linhagem SA53 de ETEC isolada de fezes de búfalo na Tailândia e pela linhagem 41 de ETEC isolada de carne crua no Brasil são consideradas toxinas LT-II padrão (GREEN et al., 1983; GUTH et al., 1986b). Embora as LT-Il sejam capazes de aumentar os níveis intracelulares de cAMP, o papel dessa classe de LT na indução de diarréia por linhagens de ETEC não está bem esclarecido (NATARO; KAPER, 1998).

Além de seu papel já estabelecido como mediadoras da diarréia, as toxinas termo-lábeis do tipo I (LT e CT) são reconhecidas por sua alta imunogenicidade e capacidade adjuvante. Essas moléculas são os melhores adjuvantes de mucosa conhecidos além de potentes agentes imunomoduladores. Relatos das propriedades imunomoduladoras de LT e CT sobre células do sistema imune (PETROVSKA et al., 
2003) e a busca por uma explicação para a capacidade adjuvante apresentada por estas proteínas quando administradas por diversas vias de inoculação (parenteral, oral, nasal, transcutânea e intradérmica) são abundantes na literatura. Acredita-se que a atividade biológica de LT ocorra graças à interação de suas subunidades $B$ com o receptor específico gangliosídeo GM1 presente na membrana de células alvo e de outras células eucarióticas. Essa hipótese baseia-se na capacidade do pentâmero $B$ isolado em induzir respostas imunes e em estudos com mutantes de LT defectivos na ligação a GM1, que não apresentam imunogenicidade ou capacidade adjuvante (GUIDRY et al., 1997;WILLIAMS; HIRST; NASHAR, 1999).

O mecanismo responsável pela atividade adjuvante de LT não é bem esclarecido, entretanto algumas reações desencadeadas pela toxina seriam capazes de explicar as propriedades adjuvantes apresentadas após administração por via de mucosa: i) aumento da permeabilidade epitelial com conseqüente elevação na captação dos antígenos co-administrados, ii) aumento na capacidade de apresentação de antígenos por várias células apresentadoras de antígeno (APCs), iii) capacidade de promover a diferenciação isotípica de células B levando a uma produção aumentada de $\lg \mathrm{A}$ e iv) efeitos estimulatórios e inibitórios complexos sobre a proliferação de células T e produção de citocinas (HOLMGREN et al., 2005). Acredita-se que o aumento na capacidade de apresentação de antígenos pelas APCs seja o fator mais importante para a atividade adjuvante (HOLMGREN et al., 2003).

Semelhante ao inicialmente demonstrado para as toxinas termo-lábeis do tipo I, as LT-II também apresentam propriedades imunomoduladoras. As atividades biológicas das toxinas LT-Il têm sido avaliadas in vitro e in vivo e os estudos realizados demonstram que essas moléculas apresentam características que as diferenciam das toxinas do grupo I e também entre si (ARCE et al., 2005; MARTIN et al., 2000; MARTIN et al., 2001). Acredita-se que os efeitos imunomoduladores apresentados pelas toxinas termo-lábeis do tipo II estão intimamente relacionados à capacidade diferencial de ligação a receptores de superfície presentes em células eucarióticas (CONNELL, 2007).

O primeiro relato de atividade imunoestimuladora de LT-II ocorreu no ano de 1998 quando Connell e colaboradores demonstraram a capacidade de LT-Ila em atuar como adjuvante pela via subcutânea em ratos, sendo capaz de induzir resposta sistêmica de anticorpos IgG anti-fimbrilina equiparáveis àquelas 
desencadeadas por CT quando empregada como adjuvante pela mesma via (CONNELL et al., 1998). O efeito adjuvante das enterotoxinas termo-lábeis do tipo II foi também demonstrado pela via intranasal em camundongos utilizando-se a adesina Agl/II de S. mutans como antígeno vacinal. Nesse estudo LT-Ila e LT-Ilb apresentaram além do efeito adjuvante sistêmico já conhecido, a capacidade de induzir resposta de mucosa, sendo encontrados anticorpos slgA na saliva e lavado vaginal dos animais imunizados (MARTIN, et al., 2000).

Arce e colaboradores (2005) demonstraram que as diferentes atividades imunomoduladoras de CT, LT-Ila e LT-Ilb podem ser parcialmente relacionadas às suas especificidades de ligação a receptores. Essas toxinas apresentam ligação diferencial a várias células do sistema imune, fato que parece relacionar-se aos níveis de gangliosídeos expressos em cada tipo celular. A toxina LT-Ila, assim como CT, é capaz de induzir apoptose em células $\mathrm{CD}^{+}$, contudo foi demonstrado que a depleção de células CD8+ por LT-lla ocorre por meio de mecanismo independente de ligação a GM1. A característica de interação a receptores distintos torna as toxinas termo-lábeis do tipo II moléculas promissoras como agentes imunomoduladores, capazes de interagir com células diferentes e de maneira diferente sobre o mesmo tipo celular.

Estudos realizados por Hajishengallis e colaboradores (2004) demonstram ainda a atividade biológica diferencial entre as holotoxinas LT-Ila e LT-Ilb em relação aos seus pentâmeros $B$ isolados (LT-IlaB e LT-IlbB). As holotoxinas não são capazes de induzir secreção de citocinas em macrófagos humanos in vitro, porém possuem importantes funções de regulação de secreção de citocinas próinflamatórias induzidas por outros compostos, como por exemplo LPS. Os pentâmeros LT-IlaB e LT-IlbB por sua vez induzem a secreção de citocinas inflamatórias nesse tipo celular (MARTIN et al., 2001). Outra atividade exclusiva dos pentâmeros das toxinas LT-II é a capacidade de interação com moléculas do sistema imune inato, conhecidas como receptores do tipo Toll (do inglês Toll-like receptors - TLRs), especificamente o TLR2 (HAJISHENGALLIS et al., 2005b).

As únicas vias de administração descritas para LT-Ila e LT-Ilb em ensaios in vivo até o presente momento são as vias subcutânea e intranasal (CONNELL et al., 1998; MARTIN et al., 2000; NAWAR et al., 2005). Os efeitos obtidos pela administração das toxinas termo-lábeis varia de acordo com a via utilizada. A administração das LTs do tipo II por outras vias de inoculação pode revelar 
propriedades ainda não descritas dessas proteínas além de gerar dados para comparação de eficiência das mesmas como adjuvantes em relação à LT-I.

A pele recobre a superfície do corpo, protege contra choques mecânicos e perda de fluidos, funciona como barreira de defesa contra patógenos e é rica em células do sistema imune, incluindo linfócitos e células apresentadoras de antígenos (APCs) como, por exemplo, as células de Langerhans e células dendríticas dermais (LEE; JEONG; AHN, 2006). Pela natureza de sua localização e presença de células importantes na geração de resposta imune, a pele torna-se um interessante sítio para administração de vacinas. O objetivo inicial do presente trabalho foi avaliar as propriedades imunomoduladoras, com ênfase na imunogenicidade e atividade adjuvante, das toxinas termo-lábeis do tipo II administradas pelas vias transcutânea e intradérmica, técnicas de imunização cutânea descritas previamente na literatura.

Ensaios de imunização transcutânea e intradérmica empregando as toxinas termo-lábeis do tipo I, CT e LT-I, como adjuvantes demonstraram a eficiência dessas moléculas em induzir respostas de anticorpos sistêmicos e secretados contra si e contra diversos antígenos (GLENN et al., 1998; GLENN et al., 1999b; SCHARTONKERSTEN et al., 2000), contudo não existem relatos na literatura do emprego das toxinas LT-Ila e LT-Ilb por estas via de inoculação. A capacidade de ligação dessas toxinas a diferentes tipos celulares pode levar a diferenças nas respostas imunes desencadeadas em comparação as toxinas LT-I (CONNELL, 2007).

A imunização transcutânea (ITC) consiste na aplicação tópica de um antígeno e um imunoestimulante (adjuvante) sobre a pele intacta e hidratada (GLENN et al., 1998), sendo capaz de desencadear uma potente resposta imune humoral sistêmica e de mucosa aos antígenos vacinais e ao adjuvante aplicados sobre a pele (PARTIDOS et al., 2003). A administração de antígenos vacinais sobre a pele torna os protocolos de imunização indolores e mais seguros aumentando a aceitação destes pela população. Além disto, a ITC e evita a transmissão de agentes patogênicos presentes no sangue como os vírus do HIV e das hepatites B e C já que não há manuseio de material pérfuro-cortante, além de reduzir custos com material estéril e treinamento de profissionais (PARTIDOS et al., 2003). A ausência de efeitos adversos de LT-I e CT quando aplicadas por ITC abre perspectivas no emprego de toxinas bacterianas nativas de forma segura em animais e seres humanos (TIERNEY et al., 2003). 
$\mathrm{Na}$ imunização intradérmica, realizada por via parenteral, a administração do antígeno e do adjuvante ocorre nas camadas superficiais da pele (epiderme e derme), sítios ricos em APCs que uma vez ativadas desempenham importante papel na maturação de linfócitos, com conseqüente geração de resposta imune de memória. Possíveis efeitos colaterais podem ser detectados facilmente pela observação visual do local de inoculação ou por cortes histológicos. O emprego das toxinas termo-lábeis do tipo I por essa via de inoculação surgiu como alternativa para potencializar a resposta imune de pacientes imunocomprometidos e idosos (ZOETEWEIJ, et al., 2006). Outra vantagem da técnica é a reduzida quantidade de antígeno e adjuvante administrados (PICOT, 2008).

Dificuldades encontradas durante o período de execução deste trabalho levaram a uma alteração do objetivo inicialmente proposto. Diante da impossibilidade de obtenção da enterotoxina LT-Ilb, por problemas na amplificação do gene alvo, optamos por avaliar as propriedades imunomoduladoras desencadeadas pela holotoxina LT-Ila e seu pentâmero B (LT-IlaB) quando administradas pelas vias transcutânea e intradérmica. Os resultados encontrados demonstram que a atividade adjuvante das toxinas dos tipos I e II varia de acordo com a via de administração empregada e que a afinidade de ligação da toxina LT-Ila a outros receptores que não GM1 pode ser responsável por diferenças na atividade inflamatória induzida por esta proteína em comparação àquela gerada pela imunização com LT-I. 


\section{CONCLUSÕES E PERSPECTIVAS}

- As proteínas LT-Ila e LT-IlaB super expressas em modelo procarioto apresentam conformação nativa e atividade biológica preservada após purificação por cromatografia em coluna niquelada.

- Diferente do observado para as toxinas do tipo I e seus pentâmeros, LT-Ila e LT-IlaB não são imunogênicas quando administrados por ITC.

- A proteína LT-Ila apresenta atividade adjuvante menor que a toxina LT-I (controle positivo) quando administrada pela via transcutânea para o antígeno OVA. O pentâmero LT-IIaB não é capaz de aumentar significativamente os níveis de anticorpos séricos contra o mesmo antígeno administrado pela via transcutânea.

- LT-Ila e LT-IlaB apresentam imunogenicidade menor que LT-I pela via intradérmica.

- As proteínas LT-Ila e LT-IlaB apresentam atividade adjuvante humoral quando administradas pela via intradérmica, contudo apenas a holotoxina é capaz de gerar resposta citotóxica em animais imunizados.

- Quando aplicadas por uma via parenteral (i.d.) as holotoxinas LT-I e LT-Ila aumentam a permeabilidade tecidual de maneira semelhante, entretanto induzem efeitos inflamatórios distintos.

- Os resultados obtidos abrem perspectivas promissoras para aplicação da toxina LT-Ila e seu pentâmero B em formulações vacinais administradas pela via intradérmica, com redução parcial ou total da reatividade inflamatória, respectivamente. 


\section{REFERÊNCIAS BIBLIOGRÁFICAS*}

ANJUÈRE, F.; GEORGE-CHANDY, A.; AUDANT, F.; ROUSSEAU, D.; HOLMGREN, J.; CZERKINSKY, C. Transcutaneous immunization with cholera toxin B subunit adjuvant suppresses lgE antibody responses via selective induction of Th1 immune responses. The Journal of Immunology, v.170, n.3, p.1586-1592, 2003.

ARCE, S.; NAWAR, H. F.; RUSSELL, M. W.; CONNELL, T. D. Differential binding of Escherichia coli enterotoxins LT-Ila and LT-IIb and of cholera toxin elicits differences in apoptosis, proliferation, and activation of lymphoid cells. Infection and Immunity, v.73, n.5, p.2718-2727, 2005.

BARBER, D. L.; WHERRY, E. J.; AHMED, R. Cutting Edge: Rapid in vivo killing by memory CD8 $T$ Cells. The Journal of Immunology, v.171, n.1, p.27-31, 2003.

BIANCHI, M. E. DAMPs, PAMPs and alarmins: all we need to know about danger. Journal of Leukocyte Biology, v, 81, p.1-5, 2007.

BIANCHI, M.E. DAMPs, PAMPs and alarmins: all we need to know about danger. Journal of Leukocyte Biology, v.81, n.1, p.1-5, 2007.

CONNELL, T. D. Cholera toxin, LT-I, LT-Ila and LT-IIb: the critical role of ganglioside binding in immunomodulation by type I and type II heat-labile enterotoxins. Expert Review of Vaccines, v.6, n.5, p.821-834, 2007.

CONNELL, T. D.; METZGER, D.; SFINTESCU, C.; EVANS, R. T. Immunostimulatory activity of LT-Ila, a type II heat-labile enterotoxin of Escherichia coli. Immunology Letters, v.62, n.2, p.117-120, 1998.

DAHLAN, A.; ALPAR, H. O.; STICKINGS, P.; SESARDIC, D.; MURDAN, S. Transcutaneous immunisation assisted by low-frequency ultrasound. International Journal of Pharmaceutics, v.368, n.1-2, p.123-128, 2008.

DE GREGORIO, E.; TRITTO, E.; RAPPUOLI, R. Alum adjuvanticity: unraveling a century old mystery. European Journal of Immunology, v.38, n.8, p.20682071, 2008.

\footnotetext{
"De acordo com:

ASSOCIAÇÃO BRASILEIRA DE NORMAS TÉCNICAS. NBR 6023: Informação e documentação: referências: elaboração. Rio de Janeiro, 2002.
} 
DE HAAN, L.; VERWEIJ, W. R.; FEIL, I. K.; HOLTROP, M.; HOL, W. G. J.; AGSTERIBBE, E., WILSCHUT, J. Role of $\mathrm{G}_{\mathrm{M} 1}$ binding in the mucosal immunogenicity and adjuvant activity of the Escherichia coli heat-labile enterotoxin and its B subunit. Immunology, v.94, n.3, p.424-430, 1998.

DELL, K.; KOESTERS, R.; GISSMANN, L. Transcutaneous immunization in mice: induction of $\mathrm{T}$-helper and cytotoxic $\mathrm{T}$ lymphocyte responses and protection against human papillomavirus-induced tumors. International Journal of Cancer, v.118, n.2, p.364-372, 2006.

DONTA, S. T.; TOMICIC, T.; HOLMES, R. K. Binding of class II Escherichia coli enterotoxins to mouse $\mathrm{Y} 1$ and intestinal cells. Infection and Immunity, v.60, n.7, p.2870-2873, 1992.

DOUGAN, G.; HORMAECHE, C. How bacteria and their products provide clues to vaccine and adjuvant development. Vaccine, n.24, Suppl.2, p.13-19, 2006.

EDELHOCH, H. Spectroscopic determination of tryptophan and tyrosine in proteins. Biochemistry, v.6, n.7, p.1948-1954, 1967.

ENIOUTINA, E. Y.; VISIC, D.; DAYNES RA. The induction of systemic and mucosal immune responses to antigen-adjuvant compositions administered into the skin: alterations in the migratory properties of dendritic cells appears to be important for stimulating mucosal immunity. Vaccine, v.18, n.24, p.2753-2767, 2000.

FREYTAG, L. C.; CLEMENTS, J. D. Mucosal adjuvants. Vaccine, v.23, n.15, p.1804-1813, 2005.

FUJIHASHI, K.; KOGA, T.; VAN GINKEL, F. W.; HAGIWARA, Y.; MCGHEE, J. R. A dilemma for mucosal vaccination: efficacy versus toxicity using enterotoxin-based adjuvants. Vaccine, v.20, n.19-20, p.2431-2438, 2002.

FUKUTA, S.; MAGNANI, J. L.; TWIDDY, E. M.; HOLMES, R. K.; GINSBURG, V. Comparison of the carbohydrate-binding specificities of cholera toxin and Escherichia coli heat-labile enterotoxins LTh-I, LT-Ila, and LT-IIb. Infection and Immunity, v.56, n.7, p.1748-1753, 1988. 
GLENN, G. M.; SCHARTON-KERSTEN, T.; VASSELL, R.; MALLETT, C. P.; HALE, T. L.; ALVING, C. R. Transcutaneous immunization with cholera toxin protects mice against lethal mucosal toxin challenge. Journal of Immunology, v.161, n.7, p.3211-3214, 1998.

GLENN, G. M.; SCHARTON-KERSTEN, T.; ALVING, C. R. Advances in vaccine delivery: transcutaneous immunization. Expert Opinion on Investigational Drugs, v.8, n.6, p.797-805, 1999a.

GLENN, G. M.; SCHARTON-KERSTEN, T.; VASSELL, R.; MATYAS, G. R.; ALVING, C. R. Transcutaneous immunization with bacterial ADP-ribosylating exotoxins as antigens and adjuvants. Infection and Immunity, v. 67, n.3, p.1100-1106, 1999b.

GLENN, G. M.; TAYLOR, D. N.; LI, X.; FRANKEL, S.; MONTEMARANO, A.; ALVING, C. R. Transcutaneous immunization: a human vaccine delivery strategy using a patch. Nature Medicine, v.6, n.12, p.1403-1406, 2000.

GREEN, B. A.; NEILL, R. J.; RUYECHAN, W. T.; HOLMES, R. K. Evidence that a new enterotoxin of Escherichia coli which activates adenylate cyclase in eucaryotic target cells is not plasmid mediated. Infection and Immunity, v.41, n.1, p.383-390, 1983.

GÜEREÑA-BURGUEÑO, F.; HALL, E. R.; TAYLOR, D. N.; CASSELS, F. J.; SCOTT, D. A.; WOLF, M. K.; ROBERTS, Z. J.; NESTEROVA, G. V.; ALVING, C. R.; GLENN, G. M. Safety and immunogenicity of a prototype enterotoxigenic Escherichia coli vaccine administered transcutaneously. Infection and Immunity, v.70, n.4, p.1874-1880, 2002.

GUIDRY, J. J.; CÁRDENAS, L.; CHENG, E.; CLEMENTS, J. D. Role of receptor binding in toxicity, immunogenicity, and adjuvanticity of Escherichia coli heat-labile enterotoxin. Infection and Immunity, v.65, n.12, p.4943-4950, 1997.

GUTH, B. E.; PICKETT, C. L.; TWIDDY, E. M.; HOLMES, R. K.; GOMES, T. A.; LIMA, A. A.; GUERRANT, R. L.; FRANCO, B. D.; TRABULSI, L. R. Production of type II heat-labile enterotoxin by Escherichia coli isolated from food and human feces. Infection and Immunity, v.54, n.2, p.587-589, 1986b.

GUTH, B. E.; TWIDDY, E. M. ; TRABULSI, L. R.; HOLMES, R. K. Variation in chemical properties and antigenic determinants among type II heat-labile 
enterotoxins of Escherichia coli. Infection and Immunity, v.54, n.2, p.529-536, 1986a.

GYLES, C.L.; PALCHAUDHURI, S.; MAAS, W.K. Naturally occurring plasmid carrying genes for enterotoxin production and drug resistance. Science, v.198, n. 4313, p.198-199, 1977.

HAJISHENGALLIS, G.; NAWAR, H.; TAPPING, R. I; RUSSELL, M. W.; CONNELL, T. D. The Type II heat-labile enterotoxins LT-Ila and LT-Ilb and their respective $B$ pentamers differentially induce and regulate cytokine production in human monocytic cells. Infection and Immunity, v.72, n.11, p.6351-6358, 2004.

HAJISHENGALLIS, G.; ARCE, S.; GOCKEL, C. M.; CONNELL, T. D.; RUSSELL, M. W. Immunomodulation with enterotoxins for the generation of secretory immunity or tolerance: applications for oral infections. Journal of Dental Research, v.84, n.12, p. 1104-1116, $2005 a$.

HAJISHENGALLIS, G.; TAPPING, R. I.; MARTIN, M. H.; NAWAR, H.; LYLE, E. A.; RUSSELL, M. W.; CONNELL, T. D. Toll-like receptor 2 mediates cellular activation by the $B$ subunits of type II heat-labile enterotoxins. Infection and Immunity, v.73, n.3, p.1343-1349, 2005b.

HOLMES, D.S.; QUIGLEY, M. A rapid boiling method for the preparation of bacterial plasmids. Analytical Biochemistry, v.114, n.1, 193-197, 1981.

HOLMES, R. K.; TWIDDY, E. M.; PICKETT, C. L. Purification and characterization of type II heat-labile enterotoxin of Escherichia coli. Infection and Immunity, v.53, n.3, p.464-473, 1986.

HOLMGREN, J.; ADAMSSON, J.; ANJUÈRE, F.; CLEMENS, J.; CZERKINSKY, C.; ERIKSSON, K.; FLACH, C. F.; GEORGE-CHANDY, A.; HARANDI, A. M.; LEBENS, M.; LEHNER, T.; LINDBLAD, M.; NYGREN, E.; RAGHAVAN, S.; SANCHEZ, J.; STANFORD, M.; SUN, J. B.; SVENNERHOLM, A. M.; TENGVALL, S. Mucosal adjuvants and anti-infection and anti-immunopathology vaccines based on cholera toxin, cholera toxin B subunit and CpG DNA. Immunology Letters, v.97, n.2, p.181-188, 2005.

KAHLON, R.; DUTZ, J. P. Skin immune responses to peptide and protein antigen are TLR4 independent. Cellular Immunology, v.226, n.2, p.116-123, 2003. 
LÁSARO, M. A. S. Diversidade genética da toxina termo-lábil de Escherichia coli enterotoxigênica (ETEC) e potenciais aplicações biotecnológicas. 159 f. Tese (Doutorado em Microbiologia) - Instituto de Ciências Biomédicas, Universidade de São Paulo, 2006.

LEE, S.H.; JEONG, S. K.; AHN, S.K. An update of the defense barrier function of skin. Yonsei Medical Journal, v.47, n.3; p.293-306, 2006.

MARTIN, M.; METZGER, D. J.; MICHALEK, S.M.; CONNELL, T. D.; RUSSELL, M. W. Comparative analysis of the mucosal adjuvanticity of the type II heatlabile enterotoxins LT-Ila and LT-IIb. Infection and Immunity, v. 68, n.1, p.281$287,2000$.

MARTIN, M.; METZGER, D.J.; MICHALEK, S. M.; CONNELL, T. D.; RUSSELL, M. W. Distinct cytokine regulation by cholera toxin and type II heat-labile toxins involves differential regulation of CD40 ligand on CD4(+) T cells. Infection and Immunity, v. 69, n.7, p.4486-4492, 2001.

MERRITT, E. A.; HOL, W. GJ. $A B_{5}$ toxins, Current Opinion in Structural Biology, v.5, n.2, p.165-171, 1995.

NASHAR, T. O.; WEBB, H. M.; EAGLESTONE, S.; WILLIAMS, N. A.; HIRST, T. $R$. Potent immunogenicity of the $B$ subunits of Escherichia coli heat-labile enterotoxin: receptor binding is essential and induces differential modulation of lymphocyte subsets. Proceedings of the National Academy of Sciences of the United States of America, v.93, n.1, p.226-230, 1996.

NASHAR, T.O.; HIRST, T. R.Immunoregulatory role of $\mathrm{H}-2$ and intra-H-2 alleles on antibody responses to recombinant preparations of B-subunits of Escherichia coli heat-labile enterotoxin ( $\mathrm{rEtxB})$ and cholera toxin ( $\mathrm{rCtxB}$ ). Vaccine, v.13, n.9, p.803-810, 1995.

NATARO, J. P.; KAPER, J. B. Diarrheagenic Escherichia coli. Clinical Microbiology Reviews, v.11, n.1, p.142-201, 1998. Published erratum appears in Clinical Microbiology Reviews, v.11, p.403, 1998.

NAWAR, H. F.; ARCE, S.; RUSSELL, M. W.; CONNELL, T. D. Mucosal adjuvant properties of mutant LT-Ila and LT-IIb enterotoxins that exhibit altered ganglioside-binding activities. Infection and Immunity, v.3, n.3, p.1330-1342, 2005. 
PARTIDOS, C. D.; BEIGNON, AS; MAWAS, F.; BELLIARD, G.; BRIAND, JP; MULLER, S. Immunity under the skin: potential application for topical delivery of vaccines. Vaccine, v. 21, p.776-780, 2003.

PETROVSKA, L.; LOPES, L.; SIMMONS, C.P.; PIZZA, M.; DOUGAN, G.; CHAIN, B. M. Modulation of dendritic cell endocytosis and antigen processing pathways by Escherichia coli heat-labile enterotoxin and mutant derivatives. Vaccine, v.28, n.13-14, p.1445-1454, 2003.

PICKETT, C. L.; TWIDDY, E. M.; COKER, C.; HOLMES, R. K. Cloning, nucleotide sequence, and hybridization studies of the type $\mathrm{Ilb}$ heat-labile enterotoxin gene of Escherichia coli. The Journal of Bacteriology, v.171, n.9, p.4945-4952, 1989.

PICKETT, C. L.; WEINSTEIN, D. L.; HOLMES, R. K. Genetics of type lla heatlabile enterotoxin of Escherichia coli: operon fusions, nucleotide sequence, and hybridization studies. The Journal of Bacteriology, v. 169, n.11, p.5180-5187, 1987.

PICOT V. Intradermal immunization: an alternative route for vaccine administration. Articles as per sessions meeting report. Vaccine, n.26, Suppl 9, p.1-5, 2008.

RAPPUOLI, R.; PIZZA, M.; DOUCE, G.; DOUGAN, G. Structure and mucosal adjuvanticity of cholera and Escherichia coli heat-labile enterotoxins. Immunology Today, v.20, n.11, p.493-500, 1999.

SACK, D. A.; HUDA, S.; NEOGI, P. K.; DANIEL, R. R.; SPIRA, W. M. Microtiter ganglioside enzyme-linked immunosorbent assay for vibrio and Escherichia coli heat-labile enterotoxins and antitoxin. Journal of Clinical Microbiology, v.11, n.1, p.35-40, 1980.

SALMOND, R. J.; LUROSS, J. A.; WILLIAMS, N. A. Immune modulation by the cholera-like enterotoxins. Expert Reviews in Molecular Medicine, v.4, n.21, p.1-16, 2002.

SAMBROOK J.; RUSSEL D. W. Molecular Cloning: A Laboratory Manual. 3rd ed. New York: Cold Spring Harbor Laboratory Press, 2001. 
SÁNCHEZ, J.; HOLMGREN, J. Virulence factors, pathogenesis and vaccine protection in cholera and ETEC diarrhea. Current Opinion in Immunology, v.17, n.4, p.388-398, 2005.

SCHARTON-KERSTEN, T.; YU, J.; VASSELL, R.; O'HAGAN, D.; ALVING, C. R.; GLENN, G. M. Transcutaneous immunization with bacterial ADPribosylating exotoxins, subunits, and unrelated adjuvants. Infection and Immunity, v.68, n.9, 5306-5313, 2000.

SERIWATANA, J.; ECHEVERRIA, P.; TAYLOR, D. N.; RASRINAUL, L.; BROWN, J. E.; PEIRIS, J. S.; CLAYTON, C. L. Type II heat-labile enterotoxinproducing Escherichia coli isolated from animals and humans. Infection and Immunity, v.56, n.5, p.1158-1161, 1988.

SMITH, H. W.; LINGGOOD, M. A. The transmissible nature of enterotoxin production in a human enteropathogenic strain of Escherichia coli. Journal of Medical Microbiology, v.4, n.3., p.301-305, 1971.

SPANGLER, B. D. Structure and function of cholera toxin and the related Escherichia coli heat-labile enterotoxin. Microbiological Reviews, v.56, n.4, p.622-647, 1992.

TAKAHASHI, I.; MARINARO, M.; KUIYONO, H.; JACKSON, R. J.; NAKAGAWA, I.; FUGIHASHI, K.; HAMADA, S.; CLEMENTS, J. D., BOST, K. B.; MCGHEE, J. R. Mechanisms for mucosal immunogenicity and adjuvanticity of Escherichia coli labile enterotoxin. The Journal of Infectious Diseases, v. 173, n.3, p.627-635, 1996.

TIERNEY, R.; BEIGNON, A. S.; RAPPUOLI, R.; MULLER, S.; SESARDIC, D.; PARTIDOS, C.D. Transcutaneous immunization with tetanus toxoid and mutants of Escherichia coli heat-labile enterotoxin as adjuvants elicits strong protective antibody responses. The Journal of Infectious Diseases, v.188, n.5, p.753-758, 2003.

UDDOWLA, S.; FREYTAG, L. C.; CLEMENTS, J. D. Effect of adjuvants and route of immunizations on the immune response to recombinant plague antigens. Vaccine, v.25, n.47, p.7984-7993, 2007.

UESAKA, Y.; OTSUKA, Y.; LIN, Z.; YAMASAKI, S.; YAMAOKA, J.; KURAZONO, H.; TAKEDA, Y. Simple method of purification of Escherichia coli heat-labile enterotoxin and cholera toxin using immobilized galactose. Microbial Pathogenesis, v.16, n.1, p.71-76, 1994. 
VAN GINKEL, F. W.; JACKSON, R. J.; YOSHINO, N.; HAGIWARA, Y.; METZGER, D. J.; CONNELL, T. D.; VU, H. L.; MARTIN, M.; FUJIHASHI, K.; MCGHEE, J. R. Enterotoxin-based mucosal adjuvants alter antigen trafficking and induce inflammatory responses in the nasal tract. Infection and Immunity, v.73, n.10, p.6892-6902.

VOGT, A.; MAHÉ, B.; COSTAGLIOLA, D.; BONDUELLE, O.; HADAM, S.; SCHAEFER, G.; SCHAEFER, H.; KATLAMA, C.; STERRY, W.; AUTRAN, B.; BLUME-PEYTAVI, U.; COMBADIERE, B. Transcutaneous anti-influenza vaccination promotes both CD4 and CD8 T cell immune responses in humans. The Journal of Immunology, v.180, n.3, p.1482-1489, 2008.

WILLIAMS, N.A.; HIRST, T.R.; NASHAR, T.O. Immune modulation by the cholera-like enterotoxins: from adjuvant to therapeutic. Immunology Today, v.20, n.2, p.95-101, 1999.

YU, J.; CASSELS, F.; SCHARTON-KERSTEN, T.; HAMMOND, S. A.; HARTMAN, A.; ANGOV, E.; CORTHÉSY, B.; ALVING, C.; GLENN, G. Transcutaneous immunization using colonization factor and heat-labile enterotoxin induces correlates of protective immunity for enterotoxigenic Escherichia coli. Infection and Immunity, v.70, n.3, p.1056-1068, 2002.

ZOETEWEIJ, J. P; EPPERSON, D. E.; PORTER, J. D.; ZHANG, C. X.; FROLOVA, O. Y.; CONSTANTINIDES, A. P.; FUHRMANN, S. R.; EL-AMINE, M.; TIAN, J. H.; ELLINGSWORTH, L. R.; GLENN, G. M. GM1 binding-deficient exotoxin is a potent noninflammatory broad spectrum intradermal immunoadjuvant. Journal of Immunology, v.177, p.1197-1207, 2006. 